\title{
Digitalization of agricultural industrial complex, application of robotics in agriculture
}

\author{
Malik Baimukhamedov*, Auzhan Baimukhamedova, Gulzada Baimukhamedova, and Murat \\ Aimurzinov,
}

Kostanay Social and Technical University, Kostanay, Kazakhstan

\begin{abstract}
The article deals with the issues of digitalization of the agroindustrial complex. It is noted that one of the important areas of digitalization is the development of information technologies and robotization of agriculture. High efficiency is achieved by those industries that effectively use robotic means - (Repair and Maintenance Stations) drones, unmanned agricultural machines (tractors, seeders, cultivators, etc.). The advantages of using RMS in agriculture are shown. Kazakhstan produces, various seeders and cultivators, combines for picking berries, selfdriving machines for spraying in fields, gardens and other RMS. Work is underway to create quadrocopters intended for use in agriculture, and the structure and mathematical model of the quadrocopter are described in the following article.
\end{abstract}

\section{Introduction}

Currently, robotics and robotic tools are being introduced into various spheres of human activity and are associated with carrying out work in uncertain and extreme conditions [1]. And today, an important direction in solving the tasks of priority development of the agroindustrial complex of the republic, solving food issues, improving the competitiveness of agricultural production is the digitalization of agro-industrial production based on automation, robotics and the development of information technologies.

Information and communication technologies are now widely used in various fields of agriculture: in crop production, animal husbandry, animal nutrition, in calculation of fertilizer doses, land management, regulation of plant nutrition and microclimate in greenhouses, etc.

High efficiency is achieved precisely by those industries that use technological complexes, where mass production and serial production are present and robotic means (RMS) are effectively used. To the following means we refer drones, unmanned agricultural machines (tractors, seeders, cultivators, etc.). With the help of automation and robotization of agricultural production, the reliability and service life of technological equipment is increased, working conditions are facilitated, and its safety is increased too. It becomes more prestigious, moreover the cost per unit of production is reduced, increasing its quantity and improving the quality of products.

\footnotetext{
*Corresponding author: bmf45@mail.ru
} 
Literature review: World practice is replete with examples of the use of RMS in agriculture. For example, the companies ISO Group, Flier Systems (the Netherlands) have robotized the processes of growing flowers in greenhouses with a system for planting them.

The product of Autonomous Tractor (SS A) was a robotic tractor without a cab. The robot of the company Blue River Technologist (USA) works as a cultivator [2]. Agrobot (Spain) produces a hydroponic system for growing and harvesting strawberries: Agrobot SW6010 and AGS Hydro.

Agribotix leases drones to cooperatives, agronomists, consultants, farm managers, and large industrial agricultural corporations. These devices allow you to take high-resolution images and maps using a variety of sensors, process them, and make maps to identify which areas of the field are most in need of fertilizer application. The company offers image processing services with a fee per unit of area for the development of maps under an annual contract [3].

These are just a few examples of the use of robotic tools in agriculture. RMSs are increasingly used in precision farming technologies that have become widespread in the last 10-15 years. These technologies made it possible to consider numerous factors affecting plant growth in a completely different way and at a different level, to reduce the cost of seeds, fertilizers, pesticides, and water, reducing the cost of production [4].

\section{Methodology}

In recent years, another wave of transformation of business and social models has been unfolding in our republic, caused by the emergence of a new generation of digital technologies, which due to the scale and depth of their influence, have been called "end-toend" - artificial intelligence, robotics, Internet of Things, wireless communication technologies, and a number of others.

One of the promising areas of development of the digital economy today is robotization, namely, the creation of robotic systems used in the management of economic systems-firms, enterprises, business structures. The state program "Digital Kazakhstan" provides for the use of RMS in all areas of public production and mainly in the field of agriculture.

The advantages of using RMS in agriculture are as follows:

- robots are able to perform various operations i.e tillage, fertilizing, sowing, planting, milking livestock, shearing wool, feeding, butchering meat and fish, etc.;

- improving business efficiency through planning, drawing up a field passport;

- increase in yield by monitoring the infestation of fields, sifting and rapid response;

- exclusion of unauthorized downtime of equipment, control of field work;

- high accuracy and speed of technological operations;

- operation in aggressive, harmful and dangerous places inaccessible to humans;

- robots monitor the cultivation of plants, track the movement of harmful insects, and allow you to make electronic maps for agriculture.

In Kazakhstan self-driving machines for spraying in fields, orchards, various seeders and cultivators, harvesters for collecting berries and other are produced today (see Figure 1 and 2). 


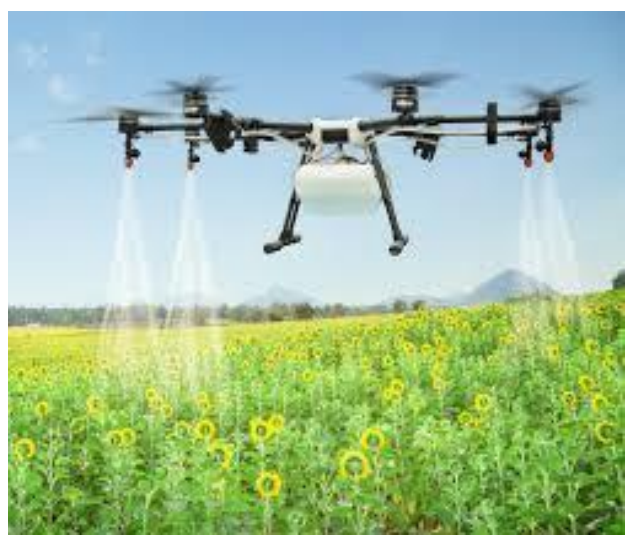

Fig. 1. Self-driving machine for spraying

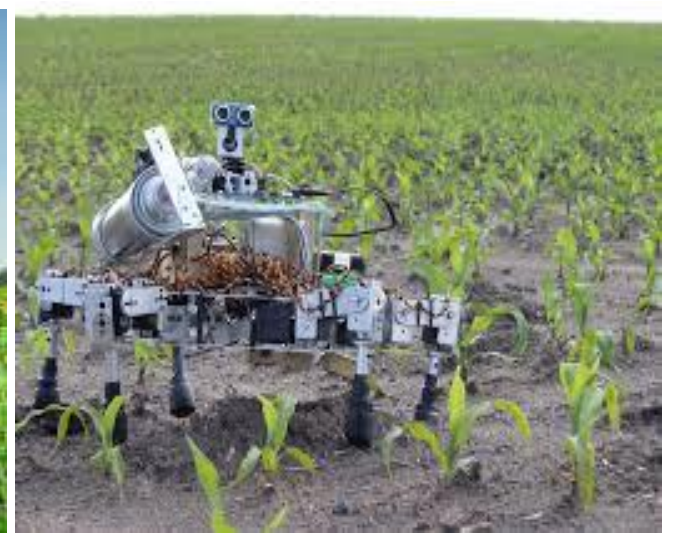

Fig. 2. Self-driving machine for cultivate

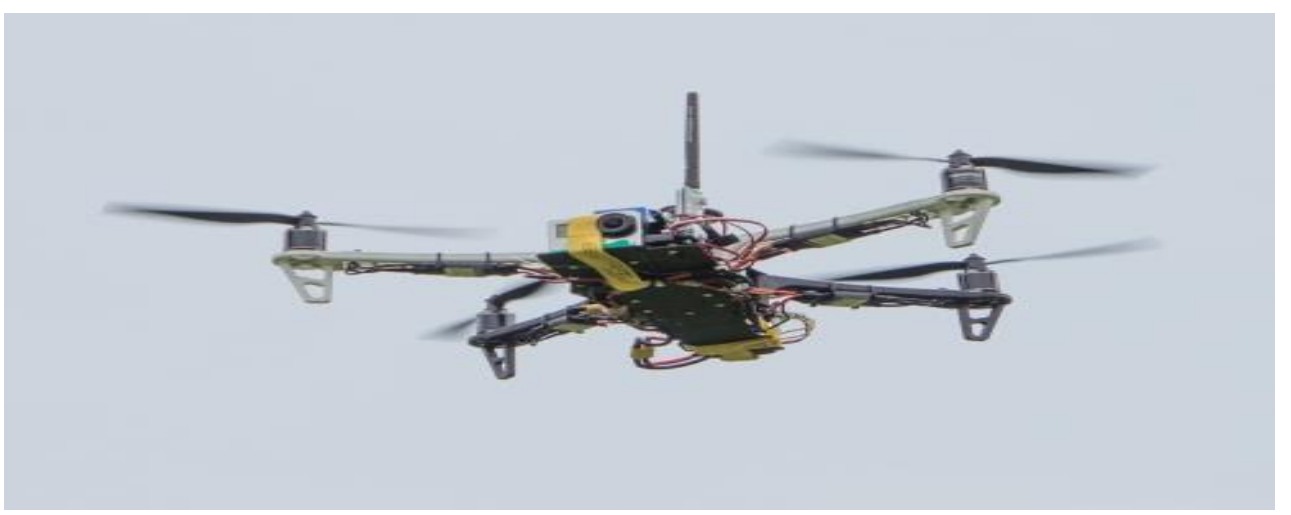

Fig. 3. Guadcopter.

In the laboratory of robotics of the Eurasian National University named after L.V. Gumilyov, research and development work is carried out to create RM and, in particular, quadrocopters intended for use in various fields, including agriculture.

Photo 3 shows a model of a quadcopter, that is kind of a sweet spot depending on weight, enhancements, installation of additional modules, payload lifting power, cost, complexity of installation, repair and flight time.

A quadcopter is a symmetrical aircraft with four equally sized rotors, propelled by

adjusting the speed of its propeller motors. The use of multiple rotors provides great maneuverability and speed and the ability to hover in the air.

They have become known as "unmanned aerial vehicles" UAVs. They offer significant advantages when used for aerial surveillance, reconnaissance and inspection in difficult and hazardous environments.

\section{Mathematical model of a quadrocopter}

The structure of a quadcopter is a rigid fixed link between four motors. The movement of the four blades causes them to direct the air flow downward, thereby causing thrust in the opposite direction. To describe a quadrocopter model, two systems of relations are usually used: $G$-system (ground), an inertial reference system is determined by the earth and $B$ system (body) - a fixed system of a body (device) (Fig. 4) [5]. The $G$-system $(O G, X G, Y G$, $Z G)$ is defined by $X G, Y G, Z G$ towards north, west and up, respectively. $B$-system $(O B, X B$, 
$Y B, Z B)$ is determined by $X B, Y B, Z B$ in the direction from the quadcopter forward, to the right and downward, respectively. The origin of this system is the center of mass of the quadcopter.

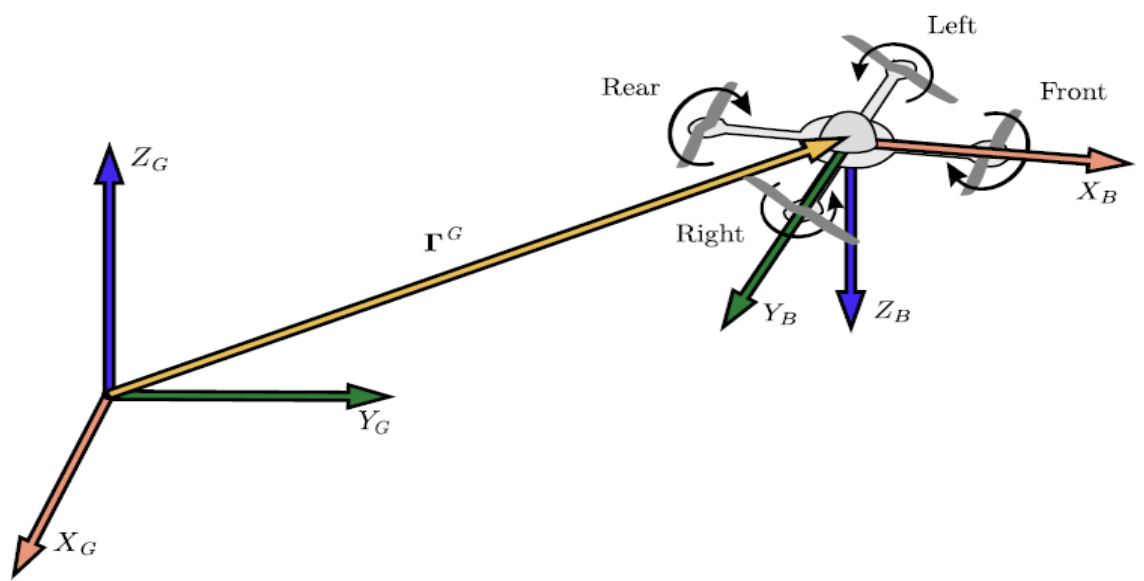

Fig.4. Graphic model of a quadcopter.

Let $\mathrm{G}$ be the position system of the quadrocopter in the coordinate system, namely, the vector of 6 degrees of freedom composed by the linear position and the angular position:

$$
\xi=(R G \Theta G)=(X, Y, Z, \phi, \theta, \psi),
$$

where $R G=(X, Y, Z)[m]$ are ordinary Cartesian coordinates, and $\Theta G=(\phi, \theta, \psi)[\mathrm{rad}]$ is the rotation of the roll, pitch, yaw axis around $X G, Y G$ and $Z G$, respectively.

Since both systems are relative to each other in a fixed coordinate system, it is possible to determine the transformation $R G$ and the rotation of the Eulerangles $(Z-Y-X)$ by the formula (1):

$$
\begin{gathered}
R_{\Theta}=R(\psi) R(\theta) R(\phi)=\left[\begin{array}{ccc}
c_{\psi} & -s_{\psi} & 0 \\
s_{\psi} & c_{\psi} & 0 \\
0 & 1 & 1
\end{array}\right]\left[\begin{array}{ccc}
c_{\theta} & 0 & s_{\theta} \\
0 & 1 & 0 \\
-s_{\theta} & 0 & c_{\theta}
\end{array}\right]\left[\begin{array}{ccc}
1 & 0 & -s_{\theta} \\
0 & c_{\phi} & c_{\theta} s_{\phi} \\
0 & -s_{\phi} & c_{\theta} c_{\theta}
\end{array}\right] \\
R_{\Theta}=\left[\begin{array}{ccc}
c_{\phi} c_{\psi}-c_{\theta} s_{\phi} s_{\psi} & -c_{\psi} s_{\phi}-c_{\phi} c_{\theta} s_{\psi} & s_{\theta} s_{\psi} \\
c_{\theta} c_{\psi} s_{\phi}+c_{\phi} s_{\psi} & c_{\phi} c_{\theta} c_{\psi}-s_{\phi} s_{\psi} & -c_{\psi} s_{\theta} \\
s_{\phi} s_{\theta} & c_{\phi} s_{\theta} & c_{\theta}
\end{array}\right]
\end{gathered}
$$

In an inertial coordinate system, the acceleration of a quadrocopter is due to thrust, gravity and linear friction. It is possible to obtain the thrust vector in an inertial coordinate system using the $\mathrm{R}$ matrix to map the thrust vector from the quadrocopter frame to the inertial coordinate system. Thus, linear motion can be obtained by the formula (2)

$$
m \vec{x}=\left[\begin{array}{c}
0 \\
0 \\
-m g
\end{array}\right]+R T_{B}+F_{D}
$$

where $\vec{x}$ is the position of the quadrocopter, $g$ is the acceleration of gravity, $F D$ is the drag force, $T B$ is the thrust vector in the quadcopter frame. You can use the center of mass of the quadrocopter as the center of reference of the inertial system to express the equation of rotation. The rotation equation can be obtained from the Euler equation for the dynamics of rigid bodies, expressed in vector form, by the formula (3) 


$$
I \dot{\omega}+\omega \times(I \omega)=\tau
$$

where $\omega$ is the vector of angular velocity, $I$ is the inertia matrix, $\tau$ is the vector of moments of external forces. (3) can be rewritten as (4):

$$
\dot{\omega}=\left[\begin{array}{c}
\dot{\omega}_{x} \\
\dot{\omega}_{y} \\
\dot{\omega}_{z}
\end{array}\right]=I^{-1}(\tau-\omega \times(I \omega)) .
$$

The quadrocopter can be modeled as two thin homogeneous rods intersecting at the origin with a point mass (motors) at the end of each. With this in mind, we can see that symmetry leads to a diagonal inertia matrix (5) of the form

$$
I=\left[\begin{array}{ccc}
I_{\mathrm{xx}} & 0 & 0 \\
0 & I_{\mathrm{yy}} & 0 \\
0 & 0 & I_{\mathrm{zz}}
\end{array}\right]
$$

Thus, you can get the final form of the mathematical model of quadrocopter in the form (6):

$$
\dot{\omega}=\left[\begin{array}{l}
\tau_{\phi} I_{x x}^{-1} \\
\tau_{\theta} I_{y y}^{-1} \\
\tau_{\psi} I_{z z}^{-1}
\end{array}\right]-\left[\begin{array}{l}
\frac{I_{y y}-I_{z z}}{I_{x x}} \omega_{y} \omega_{z} \\
\frac{I_{z z}-I_{x x}}{I_{y y}} \omega_{x} \omega_{z} \\
\frac{I_{x x}-I_{y y}}{I_{z z}} \omega_{x} \omega_{y}
\end{array}\right]
$$

This mathematical model can be used in the design of quadrocopters of various modifications.

\section{Conclusion}

The use of unmanned aerial vehicles in agriculture can become the main tool for precision farming. The desire to introduce precision farming technologies in modern agricultural enterprises leads to an increase in the efficiency of all processes. Using spectral sensors on unmanned aerial vehicles, farmers can receive information not only in the visual spectrum, but also in various spectral ranges for calculating vegetation indices or compiling soil distribution maps. All data are provided with precise coordinates with the possibility of detailed study and laboratory analysis.

Unmanned aerial vehicles used in the field of agriculture monitor plant growth, track the movement of harmful insects, and make electronic maps for agriculture. They can be roughly divided into two groups: quadrocopters and small aircraft type aircraft (drones). They differ in size, functionality, flight range, level of autonomy and other characteristics.

The main advantage of an aircraft-type UAV is its flight range. Such devices can provide a flight duration of up to three hours, while the maximum flight duration of quadrocopters used in Kazakhstani agricultural enterprises does not exceed 20-30 minutes.

Unmanned aerial vehicles and, separately, UAV services in Kazakhstan are offered by the «TerraPoint» company. The multipurpose drones offered by the company are designed for monitoring, aerial photography and mapping in agriculture and allow aerial photography from a height of 100 to 3000 meters. When acquiring ownership of a UAV, TerraPoint provides a free training service for the user of the aircraft. When an agricultural enterprise orders UAV services, TerraPoint provides services in accordance with the agreed terms of reference for the season: analysis of the NDVI vegetation index, monitoring of weediness, sifting, heterogeneity, disease development, mapping, etc. 


\section{References}

1. M.F. Baimukhamedov, A.A. Aitbenova, Fundamentals of Robotics, 189 (publishing house "Master Reprint", Kostanay, 2020)

2. B.A. Runov, Journal Agricultural machines and technologies 2, 44-52 (2016)

3. https://agrostream.net/home/article/.

4. http://www.tadviser.ru/, http://www.radixtools.ru/

5. K. Nonami, F. Fendoul, Autonomous Flying Robots 345 (Springer, 2017) 